\title{
Relaciones de la regulación emocional y la personalidad con la ansiedad y depresión en niños
}

\author{
Relations of Emotion Regulation and Personality with fnxiety \\ and Depression in Children \\ Relações da regulação emocional e a personalidade com a ansiedade \\ e depressão em crianças
}

\author{
María Laura Andrés, "Lorena Canet Juric, Claudia Elena Castañeiras, \\ María Cristina Richaud de Minzi** \\ *Universidad Nacional de Mar del Plata, Argentina. ${ }^{* *}$ Consejo Nacional de Investigaciones Cientificas \\ (Conicet), Argentina.
}

Doi: dx.doi.org/10.12804/ap134.1.2016.07

\section{Resumen}

El objetivo de este trabajo fue proponer y analizar dos modelos de asociaciones para explicar las relaciones entre los rasgos extraversión y neuroticismo; las estrategias de regulación emocional, reevaluación cognitiva (RC) y supresión de la expresión emocional (SEE), y la ansiedad y depresión en niños de 9 a 12 años de edad. Se evaluaron 230 niños de ambos géneros de dichas edades en las variables mencionadas y se contrastaron los modelos mediante análisis de senderos (path analysis). Los resultados mostraron que la RC presentó un efecto mediador total en la relación de la extraversión con la ansiedad, algo que no se observó para la depresión. La SEE presentó un efecto mediador parcial en la relación del neuroticismo con la ansiedad y con la depresión.
Estos resultados poseen valor para ampliar el conocimiento disponible respecto del efecto de estos rasgos en la sintomatología clínica, lo que resulta de utilidad para la prevención y para la planificación de tratamientos, así como para conocer, en este sentido, el efecto mediador de dichas estrategias - que son modificables o entrenables - en los rasgos de personalidad, lo que constituye un aporte para el diseño y contenido de programas de salud mental infantil.

Palabras clave: regulación emocional; personalidad; ansiedad; depresión; niños.

\section{fbstract}

The aim of this study was to propose and analyze two models of associations to explain the relationships

* María Laura Andrés, Lorena Canet Juric y Claudia Elena Castañeiras, Centro de Investigaciones en Metodología, Educación y Procesos Básicos, Universidad Nacional de Mar del Plata, Argentina.

** María Cristina Richaud de Minzi, Centro Interdisciplinario de Investigaciones en Psicología Matemática y Experimental del Consejo Nacional de Investigaciones Científicas y Técnicas (Conicet), Argentina.

La correspondencia relacionada con este artículo debe enviarse a María Laura Andrés, Funes 3250-Cuerpo V-nivel III, Mar del Plata (7600), Buenos Aires, Argentina. Correo electrónico: marialauraandres@gmail.com

Cómo citar este artículo: Andrés, M. L., Canet, L., Castañeiras, C. E. \& Richaud, M. C. (2016). Relaciones de la regulación emocional y la personalidad con la ansiedad y depresión en niños. Avances en Psicología Latinoamericana, 34(1), 99-115. doi: dx.doi.org/10.12804/apl34.1.2016.07 
between Extraversion and Neuroticism traits, emotional regulation strategies cognitive reappraisal (CR) and suppression of emotional expression (SEE) and anxiety and depression in children 9 to 12 years old. For this, 230 children of both genders of those ages were evaluated in these variables and the models were compared by Path Analysis. The results showed that CR presented a total mediating effect on the relationship of Extraversion with anxiety, which was not observed for depression. The SEE submitted a partial mediating effect on the relationship of Neuroticism with anxiety and depression. These results have value to expand the knowledge available about the effect of these traits on clinical symptomatology which is useful for the prevention and treatment planning as well as for such an effect is through strategies that are themselves modifiable and/ or trainable what constitutes an contribution for the design and content of children's mental health programs. Keywords: emotional regulation; personality; anxiety; depression; children.

\section{Resumo}

O objetivo deste trabalho foi propor e analisar os modelos de associações para explicar as relações entre os rasgos Extroversão e Neuroticismo, as estratégias de regulação emocional reavaliação cognitiva (RC) e suspensão da expressão emocional (SEE) e a ansiedade e depressão em crianças de 9 a 12 anos de idade. Avaliaram-se 230 crianças de ambos os gêneros de ditas idades nas variáveis mencionadas e se contrastaram os modelos mediante Análise de Caminhos (Path Analysis). Os resultados mostraram que a $\mathrm{RC}$ apresentou $\mathrm{m}$ efeito mediador total na relação da Extroversão com a ansiedade, coisa que não foi observada para a depressão. A SEE apresentou um efeito mediador parcial na relação do Neuroticismo com a ansiedade e com a depressão. Estes resultados possuem valor para ampliar o conhecimento disponível respeito do efeito destes rasgos sobre a sintomatologia clínica o que resulta de utilidade para a prevenção e planificação de tratamentos, assim como para conhecer que dito efeito é através de estratégias que são em si mesmas modificáveis e/ou treináveis o que constitui um impacto para o desenho e conteúdo de programas de saúde mental infantil.

Palavras-chave: regulação emocional; personalidade; ansiedade; depressão; crianças.

La ansiedad y la depresión son respuestas afectivas que sirven a importantes funciones de adaptación (MacKinnon \& Hoehn-Saric, 2003). Sin embargo, la recurrencia, la persistencia y la intensidad de estas respuestas pueden dificultar el funcionamiento psicosocial y fisiológico del individuo (Goodyer \& Sharp, 2005). En los niños, la ansiedad o la depresión en niveles elevados acarrea dificultades en su rendimiento escolar y en las relaciones interpersonales y sociales (Casey, 1996).

Se han propuesto varios factores para explicar la presencia de síntomas de ansiedad y depresión (e. g. Bragado, Bersabé \& Carrasco, 1999), entre ellos los rasgos de personalidad (Bolger \& Zuckerman, 1995). Los rasgos de personalidad definen una tendencia global y general a experimentar la vida de un modo predominantemente positivo o negativo (Diener, 1984). Se ha indicado que los altos niveles de afectividad negativa, característicos del neuroticismo, están presentes en varios trastornos emocionales y que la menor presencia de emociones positivas, sociabilidad y actividad, características de las bajas puntuaciones en extraversión, se encuentran principalmente en los trastornos relacionados con depresión y ansiedad (Bienvenu et al., 2004; Brown, 2007; Brown, Chorpita \& Barlow, 1998; Lahey, 2009).

En trabajos con niños y adolescentes, existe evidencia de que la extraversión y el neuroticismo se asocian con la presencia de síntomas clínicos. Al respecto, Muris, de Jong y Engelen (2004) y Meesters, Muris y van Rooijen (2007) encontraron en niños y adolescentes de 8 a 13 años de edad y de 9 a 17 años de edad, respectivamente, una asociación positiva entre el neuroticismo y la ansiedad. Vreeke y Muris (2002) hallaron que los padres de niños y adolescentes de 6 a 13 años de edad con 
trastornos de ansiedad evaluaron a sus hijos como más neuróticos y menos extravertidos que los padres de los niños del grupo control. En relación con la depresión, Agha-Yousefi y Maleki (2011) encontraron en una muestra de niños de 6 a 12 años de edad que el neuroticismo, en sentido positivo, y la extraversión, en sentido negativo, explicaron el $26 \%$ de la varianza de la sintomatología depresiva. En cuanto a estudios que exploraron ambos rasgos y la presencia de ambos tipos de síntomas (ansiedad y depresión), Ehrler y Evans (1999) hallaron que los niños de 9 a 13 años de edad con puntuaciones bajas en extraversión y elevadas en neuroticismo presentaban mayor cantidad tanto de síntomas de ansiedad como de depresión. Sin embargo, John, Caspi, Robins, Moffitt y Stouthamer-Loeber (1994) encontraron que los niños y adolescentes de entre 12 y 13 años de edad que sus padres describieron como más neuróticos presentaron mayor cantidad de síntomas internalizantes, en tanto que la extraversión no mostró ningún tipo de asociación.

Si bien la evidencia parece ser consistente respecto de que el neuroticismo y la extraversión se relacionan con la presencia de síntomas de ansiedad y depresión, aún se requieren nuevos estudios que puedan aportar más evidencia respecto al rol específico de los rasgos de personalidad, y queda sin resolver mediante cuáles procesos e mecanismos la personalidad ejercería su influencia en estos indicadores clínicos de ansiedad y depresión (Bolger \& Zuckerman, 1995).

Otro factor asociado a la sintomatología de ansiedad y depresión consiste en la forma en la que los individuos responden a la emoción generada por diferentes eventos. En efecto, en los últimos años han proliferado trabajos que muestran cómo las estrategias de regulación emocional se relacionan con las respuestas de ansiedad y depresión en niños y adolescentes y se ha comenzado a aportar evidencia de que podrían constituir los procesos mediante los cuales la personalidad ejerce su influencia en estas respuestas (e.g. Yoon, Maltby \& Joorman, 2013). La regulación emocional puede definirse como "todos aquellos procesos intrínsecos y extrínsecos responsables del monitoreo, evaluación y modificación de las reacciones emocionales, especialmente sus características de intensidad y duración, en orden a cumplimentar con los objetivos individuales" (Thompson, 1994, p. 24).

El modelo de proceso de regulación emocional de Gross y Thompson (2007) postula que las estrategias de regulación se diferencian según el momento en el que tienen su primer impacto en el despliegue temporal de la secuencia emocional. Las estrategias "focalizadas en el antecedente" son aquellas que sobrevienen antes de que las tendencias de respuesta emocional estén completamente activadas y las estrategias "focalizadas en la respuesta" son aquellas que se implementan cuando la respuesta emocional ya se ha generado. Dentro de este modelo, las estrategias de reevaluación cognitiva (RC) y de supresión de la expresión emocional (SEE) han recibido mayor investigación empírica y experimental. La RC es una estrategia focalizada en el antecedente que se refiere a la construcción de significados alternativos a un evento con el objeto de disminuir el impacto emocional negativo. La SEE es una estrategia focalizada en la respuesta destinada a inhibir la expresión de la emoción (Gross \& Thompson, 2007).

Si bien es poco probable que una estrategia resulte siempre adaptativa o siempre disfuncional, la investigación con adultos ha mostrado que el uso frecuente de la $\mathrm{RC}$ puede prevenir o reducir la experiencia de afecto negativo, en tanto que el uso frecuente de la SEE no modifica la experiencia de afecto negativo e incluso puede incrementarla (Gross \& John, 2003; John \& Gross, 2004). En niños y adolescentes se ha observado un patrón similar. Por ejemplo, Hughes, Gullone, Dudley y Tonge (2010) encontraron que participantes con síntomas de rechazo escolar de 10 a 14 años edad informaron menor uso de la RC y mayor uso de la SEE, que el grupo control. Gullone y Taffe (2011) mostraron que la RC en sentido negativo y la SEE en sentido positivo se asociaron con síntomas de depresión en niños y adolescentes de 10 a 18 años de 
edad. Garnefski et al. (2007) hallaron evidencia de que la RC se asoció negativamente con síntomas de ansiedad y depresión en niños de 9 a 11 años de edad. Por su parte, Penza-Clyve y Zeman (2002) encontraron que la SEE se asoció con síntomas internalizantes en niños de entre 9 y 12 años de edad.

Si bien la mayoría de dichos estudios indican que la RC posee un patrón de asociaciones más saludable, en tanto que la SEE posee un patrón relacionado con la sintomatología, se ha sugerido que estos trabajos no deberían concluir respecto de la capacidad predictiva de la regulación emocional en la psicopatología sin tener en cuenta la reactividad emocional de cada individuo que puede estar contribuyendo a explicar las diferencias individuales en la propensión a experimentar ansiedad y depresión de manera recurrente, persistente e intensa, así como en la capacidad para implementar de manera efectiva estrategias de regulación emocional (Lewis, Zinbarg \& Durbin, 2010). En este sentido, se ha indicado que la experiencia de emociones positivas característica de la extraversión puede favorecer la flexibilidad del pensamiento y la búsqueda de nuevas perspectivas característica de la RC (Carver \& Connor Smith, 2010; Isen, 1990). Gullone y Taffe (2011) y Gresham y Gullone (2012) hallaron que la extraversión se asoció positivamente con la RC. Por otro lado, la mayor experiencia de emociones negativas, de autocrítica, de sensibilidad a la crítica de otros y de sentimientos de inadecuación personal propios del neuroticismo (Watson, Clark \& Harkness, 1994) puede predisponer a los individuos a realizar mayores intentos por suprimir la expresión de sus sentimientos.

La expresión de las emociones negativas de estos individuos puede generar rechazo y malestar en las relaciones interpersonales - noción que ha sido conceptualizada por las teorías interpersonales de la depresión (e.g. Coyne, 1976)—, lo que podría favorecer que intenten inhibir el curso expresivo comportamental de sus emociones negativas, con el objetivo de salvaguardar los vínculos e interacciones personales. Los estudios mencionados de Gullone y Taffe (2011) y Gresham y Gullone (2012) hallaron que el neuroticismo se asoció positivamente con la SEE. Por lo tanto, tomar en cuenta el efecto de los rasgos de personalidad, que se caracterizan por la experiencia de emociones positivas (tal como la extraversión) o de emociones negativas (como el caso del neuroticismo), puede aportar información que otorgue mayor validez a las relaciones entre las estrategias de regulación emocional y los indicadores de psicopatología.

En síntesis, al considerar la necesidad de contar con mayor evidencia respecto de las relaciones de la personalidad con la psicopatología, así como de proponer mecanismos que expliquen la influencia de los rasgos de personalidad sobre la ansiedad y depresión - tomando en cuenta los posibles efectos de los aspectos más estables de los individuos sobre las estrategias de regulación emocional que a su vez influyen en la sintomatología clínica- se plantea como objetivo de este trabajo proponer y analizar dos modelos de asociaciones que permitan explicar las relaciones de los rasgos de personalidad extraversión y neuroticismo con las estrategias de regulación emocional RC y SEE y con los indicadores clínicos de ansiedad (modelo de ansiedad) y depresión (modelo de depresión). Específicamente, sería esperable que la extraversión se asocie negativamente con la ansiedad y depresión a través de la $\mathrm{RC}$, y que el neuroticismo se asocie positivamente con la ansiedad y depresión a través de la SEE. Si bien existen escasos estudios que han analizado el efecto mediador de la regulación emocional en estas relaciones, los resultados parecen indicar que efectivamente esta tendría un rol mediador. Por ejemplo, Ng y Diener (2009) hallaron que la estrategia de regulación emocional "saborear" - que consiste en mantener y extender las emociones positivas - presentaba un efecto mediador en la relación entre la extraversión y la tendencia a experimentar emociones positivas; en tanto que la estrategia de "reparación del ánimo" mediaba la relación del neuroticismo con la tendencia a experimentar emociones negativas. Wang, Shi \& 
Li (2009) hallaron que la extraversión y el neuroticismo influyen en el afecto positivo y negativo, respectivamente, a través de la $\mathrm{RC}$; sin embargo, no encontraron el mismo efecto mediador para la SEE. El estudio de Yoon et al. (2013) aporta evidencia sobre el efecto mediador de la SEE en la relación del neuroticismo con los síntomas depresivos pero no así con la RC.

Estos estudios aportan datos respecto del rol de la regulación emocional en la relación entre los rasgos de personalidad y la presencia de sintomatología; sin embargo, son necesarios nuevos estudios para determinar su efecto mediador (Wang et al., 2009). Por otro lado, estos resultados se refieren exclusivamente a población adulta. Si bien es sabido que las dificultades funcionales y psicopatológicas en niños y adolescentes no siempre conllevan dificultades en su adultez, sí se ha registrado que las dificultades de los adultos casi siempre devienen de dificultades más tempranas (Garnefski, Legerstee, Kraaij, van den Kommer, \& Teerds, 2002; Merikangas \& Angst, 1995; Smith, 1995), por lo que resulta valioso analizar estas relaciones en la población infantil. Además, si la influencia de los aspectos más estables de los individuos, como lo son los rasgos de personalidad, sobre la presencia de indiciadores clínicos se realiza a través de estrategias que son en sí mismas modificables o entrenables (e.g. Baumeister, Gailliot, DeWall \& Oaten, 2006; MacLeod, Koster \& Fox, 2009), estos resultados serían valiosos para el diseño de programas de promoción de la salud mental infantil (Garnefski et al., 2007).

\section{Metodología}

\section{Participantes}

Se obtuvo una muestra intencional no probabilística de 230 niños, de ambos géneros (110 varones y 120 mujeres $)$, de $9(n=65), 10(n=53), 11$ ( $n=$ $45)$ y $12(n=67)$ años de edad, provenientes de la ciudad de Mar del Plata (Argentina), que estaban cursando cuarto, quinto y sexto año de la educación primaria básica y primer año de la educación secundaria, respectivamente. La media de edad fue de 11.04 años ( $D E=1$ año y 2 meses).

El nivel socioeducativo (NSE) se calculó utilizando el Índice de Hollingshead (2011). Primero se clasificó el nivel educativo según la escala de Pascual, Galperín y Bornstein (1993), que toma como referencia el sistema educativo argentino y luego el nivel ocupacional, según la Escala de Grupos Ocupacionales de Argentina EGO 70 de Sautú (1989). Ambas escalas poseen una correlación de .94 con el Índice de Hollingshead (Pascual et al., 1993). El 18.79\% de las familias poseía NSE medio-alto; el $49.10 \%$, medio, y el $31.20 \%$ restante NSE, medio-bajo.

\section{Instrumentos de medida}

Cuestionario Argentino de Personalidad Infantil (CAPI). Para evaluar los rasgos de personalidad se utilizaron las subescalas de extraversión y neuroticismo del CAPI de Lemos (2006), basado en el modelo del NEOPI-R de Costa y McCrae (Costa, McCrae \& Martín, 2008), que operacionaliza el modelo de personalidad de los Cinco Grandes. Las subescalas de extraversión y neuroticismo se componen de 14 y 8 ítems, respectivamente, en formato escala tipo Likert de tres opciones ("Sí", "A veces", "No"). Por ejemplo: "Me divierto con facilidad" (extraversión); "Me pongo muy nervioso si la maestra me hace pasar al frente" (neuroticismo). Los análisis factoriales del instrumento realizados por la propia autora indicaron una estructura conformada por los cinco grandes factores propuestos por Costa y McCrae (1992) que explicaron el 55\% de la varianza. Los coeficientes de fiabilidad fueron desde .37 hasta .71 . Los resultados psicométricos básicos de los ítems y dimensiones resultantes fueron satisfactorios. Los valores alfa de Cronbach obtenidos en nuestra muestra fueron de .56 para extraversión y de .73 para neuroticismo. 


\section{Cuestionario de Regulación Cognitiva de la} Emoción para Niños (CERQ-k). Para la evaluación de la estrategia RC se utilizó la subescala de RC del CERQ-k, de Garnefski, et al. (2007). Consiste en una subescala de 4 ítems con opciones de respuesta en formato tipo Likert desde "Nunca" hasta "Siempre" y contiene ítems que implican asignar un significado positivo a los eventos experimentados; por ejemplo: "Pienso que lo que pasó también tiene sus cosas buenas", "Pienso que puedo aprender algo de lo que me pasó". El estudio de las propiedades psicométricas de la versión original de Garnefski et al. (2007) mostró la existencia de nueve factores que explicaron el 57.6\% de la varianza, coeficientes de fiabilidad desde .67 hasta .79 y relaciones con depresión, preocupación y temor. Este instrumento se tradujo y puso a prueba — con autorización de las autoras (N. Garnefski, comunicación personal, 9 de mayo de 2009) - en una prueba piloto de 60 niños escolarizados de 10 años de edad. El valor alfa de Cronbach para la subescala $\mathrm{RC}$ en nuestra muestra fue de .61 .

Escala de Expresividad Emocional Infantil (EESC). Para evaluar la SEE se utilizó la subescala de Reticencia a Expresar Emociones Negativas de la EESC, de Penza-Clyve y Zeman (2002). Se compone de 8 ítems con opciones de respuestas en formato tipo Likert desde "Nunca" hasta "Siempre" e indaga por la renuencia a expresar emociones negativas. Ejemplos de ítems son: "Prefiero ocultar o esconder mis sentimientos"; "Cuando estoy molesto/a, me cuesta mostrar que me siento así”. Los análisis de las propiedades psicométricas del instrumento realizados por las autoras indicaron un alfa de .81 para esta subescala y validez convergente con medidas de ansiedad, depresión y somatización, entre otras. Este instrumento se tradujo y puso a prueba - con autorización de las autoras (J. Zeman, comunicación personal, 17 de octubre de 2011) — en una prueba piloto de 54 niños escolarizados de 9 a 11 años de edad. El valor alfa de Cronbach para la subescala SEE en nuestra muestra fue de 67.

\section{Cuestionario de Autoevaluación Ansiedad} Estado/Rasgo en Niños (STAIC). Para evaluar la ansiedad se utilizó la subescala de Ansiedad Rasgo del STAIC, de Spielberger (1973) validado y adaptado a población española por Seisdedos Cubero (1998). La subescala contiene 20 ítems con opciones de respuesta tipo Likert que van desde "Casi Nunca" a "Casi siempre" e indaga la tendencia a experimentar ansiedad, por ejemplo: "Me siento desgraciado". Los análisis de fiabilidad de la adaptación española son consistentes y comparables a los obtenidos en los estudios americanos de la versión original del instrumento (Spielberger, 1973). Se introdujeron algunas variaciones en las expresiones de la versión española, adecuándola a frases y palabras que fueran más cotidianas y de uso frecuente en nuestra población. Por ejemplo, el ítem 3 de la versión española "Me siento desgraciado", se cambió por "Siento que tengo mala suerte". El valor alfa de Cronbach para la subescala ansiedad rasgo en nuestra muestra fue de .77 .

\section{Escala de Autoinforme de Sintomatología} Depresiva para Niños y Adolescentes (DDPCA). Para evaluar la depresión se utilizó la DDPCA, de Harter y Nowakowski (1987), validada y adaptada para población argentina por Richaud de Minzi, Sacchi y Moreno (2001). Cuenta con 12 ítems y escala de respuesta tipo Likert desde "No me parezco" hasta "Sí, me parezco". Evalúa el estado de ánimo, la autovaloración general, el nivel de energía y la culpabilización, por ejemplo: "Algunos chicos están tristes la mayor parte del tiempo". El análisis factorial exploratorio de la adaptación de Richaud de Minzi et al. (2001), a nuestro contexto, permitió observar las dimensiones propuestas por Harter y Nowakowski (1987) y los alfas de Cronbach presentaron valores adecuados desde .71 hasta .76 . El valor alfa de Cronbach para la escala de síntomas de depresión en nuestra muestra fue de .69.

Encuesta semiestructurada. Se envió a los padres de los niños una encuesta semiestructurada 
en la que se les pidió información relativa al nivel educativo y tipo de ocupación de cada progenitor.

\section{Procedimiento de recolección de los datos y consideraciones éticas}

Una vez que se les informó a los directivos de las instituciones educativas de los objetivos del estudio y dieron su permiso, se solicitó el consentimiento informado de los padres de los niños a través de una nota en la que se explicó el objetivo, el procedimiento y la finalidad de la investigación, y se ofreció una breve descripción de los instrumentos, así como el aseguramiento de que la participación era voluntaria y anónima. Luego se informó a los niños del estudio y se evaluó a aquellos que dieron su asentimiento para participar. Se entrevistaron en horario escolar, en pequeños grupos de no más de cinco niños por vez, en un aula que la institución educativa destinó para tal fin. La administración se realizó en un encuentro de 30 minutos aproximadamente.

\section{Plan de análisis de los datos}

Para contrastar el modelo de ansiedad y el modelo de depresión se utilizó análisis de senderos (path analysis). Se utilizó el Paquete Estadístico para Ciencias Sociales (SPSS) (v. IBM SPSS Statistics 15) y el programa Análisis de Estructuras Momentáneas (AMOS) (v. IBM SPSS AMOS 19).

Antes de realizar los modelos se realizó la preparación de los datos (Kline, 2011). Se utilizó el método de máxima verosimilitud para la estimación de los modelos y los indicadores y criterios más frecuentes para el diagnóstico de la bondad de ajuste. Para evaluar la significación de los efectos directos, indirectos y totales se utilizó el método de remuestreo bootstrap (MacKinnon, Lockwoo \& Williams, 2004) con 1000 muestras seleccionadas al azar del conjunto total de los datos e intervalos de confianza corregidos (CI) del 95\%. Si los CI no incluyen el cero y los valores $p<.05$ los efectos se consideran estadísticamente significativos (Kline, 2011).

\section{Resultados}

\section{Preparación de los datos}

Como primer paso, se exploró y descartó la presencia de colinealidad entre las variables $\left(\mathrm{R}^{2}>.90\right.$ en todos los casos). En segundo lugar, se analizó la proporción de datos faltantes en cada una de las variables. En todas, la proporción fue menor al 5\%. Luego, se utilizó el método de expectación-maximación para reemplazar los valores faltantes. El análisis de los valores de asimetría y curtosis resultó satisfactorio, ya que todos los valores se ubicaron entre 1 y-1 (George \& Mallery, 2011), y el coeficiente de curtosis multivariante de Mardia (1970) presentó un CR de -.376, lo que indicaría una distribución normal de los datos. No se detectaron casos con desviaciones mayores a 3.26 $D E$ respecto de la media en alguna o más de las variables (Tabachnick \& Fidell, 2001). Se exploró la presencia de casos atípicos multivariados mediante el método del cuadrado de la distancia de Mahalanobis y no se detectaron casos con valores de significación menores a .001.

\section{Análisis descriptivos}

En la tabla 1 se presentan las correlaciones, los estadísticos descriptivos y los valores de confiabilidad de los rasgos de personalidad, estrategias de regulación emocional y ansiedad y depresión.

La extraversión presentó correlaciones solamente con la RC y el neuroticismo con SEE, ansiedad y depresión. Las estrategias de regulación emocional presentaron asociaciones con los síntomas clínicos en los sentidos esperados, es decir, la RC en sentido negativo y la SEE en sentido positivo.

\section{Estimación de los modelos}

Las variables de personalidad y de regulación emocional explicaron el $40 \%$ de la varianza en el modelo de ansiedad (figura 1), y el $24 \%$ en el 
modelo de depresión (figura 2). Los estadísticos de bondad de ajuste y medidas de error presentaron valores dentro de lo sugerido por la bibliografía para indicar un buen o adecuado ajuste (Bentler, 1995) (tabla 2).

En términos generales, ambos modelos mostraron que la regulación emocional posee un efecto mediador en la relación de los rasgos de personalidad con la sintomatología clínica (tabla 3). Específicamente, la RC presentó un efecto mediador total en la relación de la extraversión con la ansiedad, algo que no se observó para la depresión. Si bien en el modelo de depresión se observa una asociación positiva de la extraversión con la $\mathrm{RC}-\mathrm{y}$ en los análisis de correlaciones bivariadas, una asociación negativa entre la RC y la depresión- esta estrategia no presenta un efecto mediador al ser introducida en el modelo simultáneamente con la SEE. Por su parte, la SEE presentó un efecto mediador parcial en la relación del neuroticismo con la ansiedad y con la depresión. El neuroticismo mostró un efecto directo sobre la ansiedad y la depresión en ambos modelos.

Tabla 1

Resumen de las correlaciones, media, desvio estándar y valores de confiabilidad para las variables bajo estudio

\begin{tabular}{lcccccccccc}
\hline & 2 & 3 & 4 & 5 & 6 & Mín. & Máx. & M & $D E$ & $\alpha$ \\
\hline 1. Extraversión & .01 & $.28^{* *}$ & -.08 & -.03 & -.01 & 1.33 & 3.00 & 2.41 & .350 & .56 \\
2. Neuroticismo & & -.08 & $.31^{* *}$ & $.52^{* *}$ & $.39^{* *}$ & 1.06 & 2.95 & 2.16 & .371 & .73 \\
3. RC & & & $-.20^{* *}$ & $-.22^{* *}$ & $-.13^{*}$ & 4.00 & 20 & 14.80 & 3.31 & .61 \\
4. SEE & & & & $.49 * *$ & $.41^{* *}$ & 8.00 & 38.00 & 22.33 & 5.64 & .67 \\
5. Ansiedad & & & & & $.47^{* *}$ & 21.00 & 54.00 & 37.19 & 6.17 & .77 \\
6. Depresión & & & & & 12.00 & 32.00 & 20.16 & 4.13 & .69 \\
\hline
\end{tabular}

$* p<.05 ; * * p<.01 ; * * *<.001$

RC: reevaluación cognitiva; SEE: supresión de la expresión emocional.

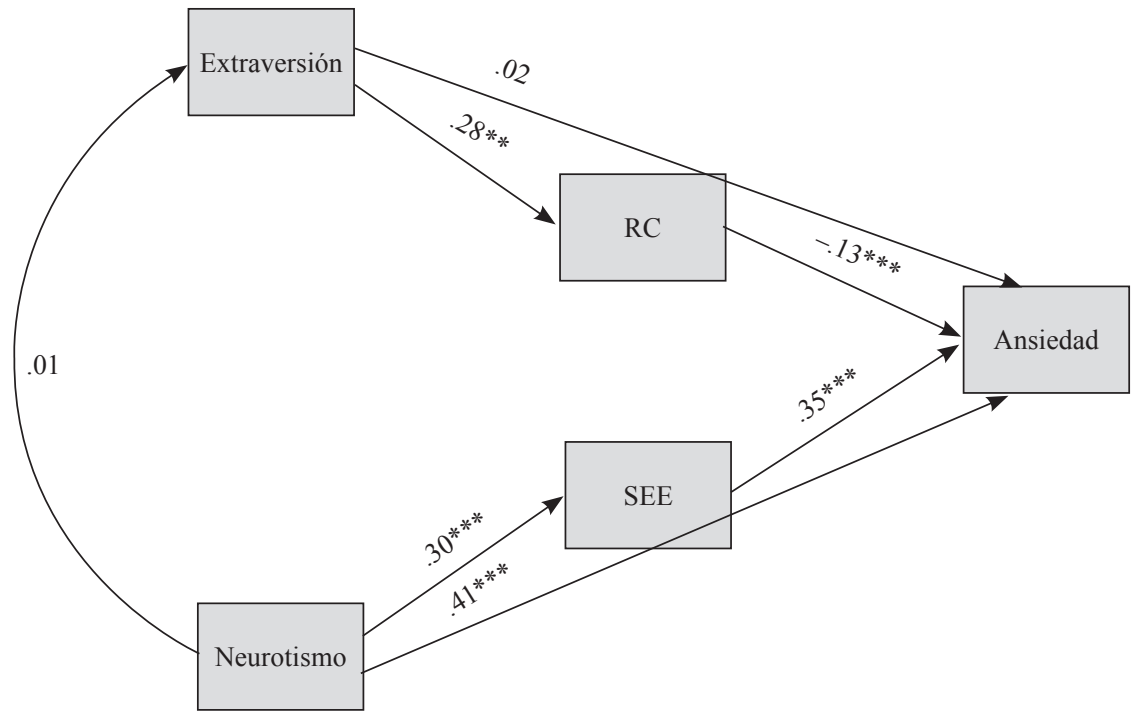

Figura 1. Coeficientes estandarizados para el modelo de ecuaciones estructurales de ansiedad

$\mathrm{RC}$ : reevaluación cognitiva; SEE: supresión de la expresión emocional.

${ }^{*} p<.05 ; * * p<.01 ; * * * p<.001$ 


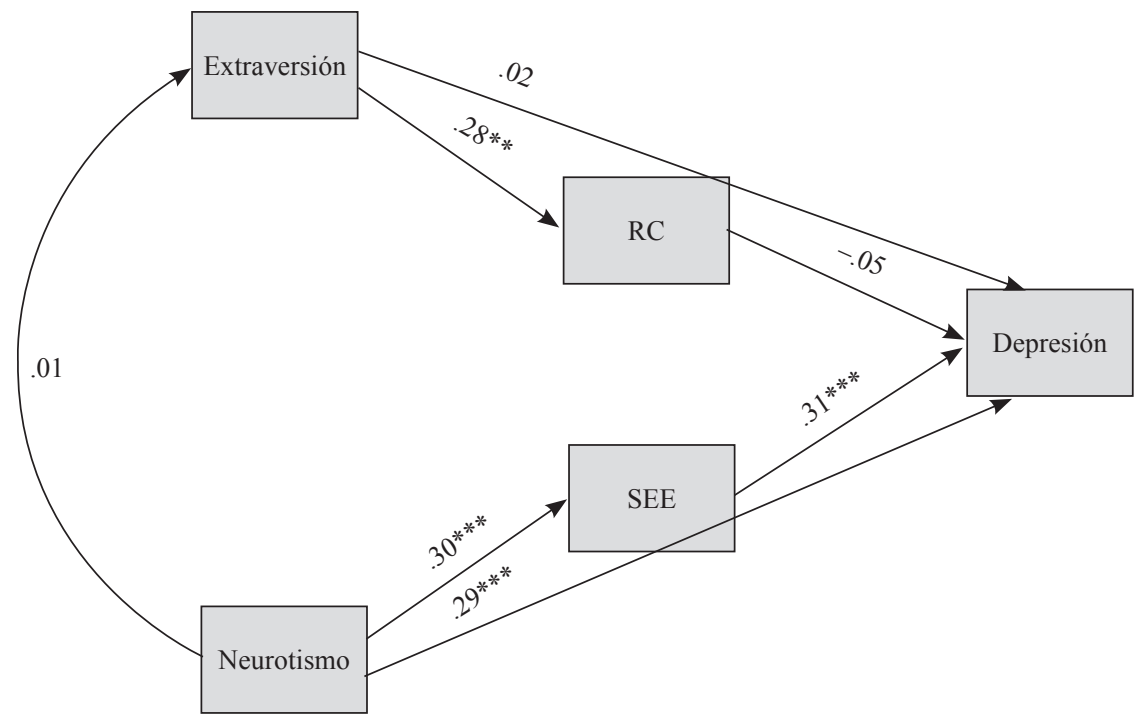

Figura 2. Coeficientes estandarizados para el modelo de ecuaciones estructurales de depresión

$\mathrm{RC}$ : reevaluación cognitiva; SEE: supresión de la expresión emocional.

$* p<.05 ; * * p<.01 ; * * * p<.001$

Tabla 2

Indicadores de ajuste del modelo de ansiedad y del modelo de depresión

\begin{tabular}{cccccccccc}
\hline Modelo & $\chi^{2}$ & Df & $p$ & CMIN/DF & GFI & CFI & RMSEA & $\begin{array}{c}90 \% \text { CI para } \\
\text { RMSEA }\end{array}$ & $\begin{array}{c}\text { RMR } \\
\text { estandarizado }\end{array}$ \\
\hline Ansiedad & 1.80 & 1 & .179 & 1.80 & .99 & .99 & .05 & $00-.19$ & .025 \\
Depresión & 1.80 & 1 & .178 & 1.80 & .99 & .99 & .05 & $00-.19$ & .024 \\
\hline
\end{tabular}

Tabla 3

Efectos totales, directos e indirectos estandarizados de los rasgos de personalidad sobre la ansiedad y la depresión

\begin{tabular}{ccccc}
\hline & Modelo & Efecto total & Efecto directo & Efecto Indirecto total \\
\hline \multirow{2}{*}{ Ansiedad } & Extraversión & -.015 & .021 & $-.037^{*}$ \\
& Neuroticismo & $.510^{* *}$ & $.40^{* *}$ & $.104^{* * *}$ \\
\multirow{2}{*}{ Depresión } & Extraversión & .005 & .020 & -.015 \\
& Neuroticismo & $.386^{* *}$ & $.293^{* *}$ & $.093^{* * *}$ \\
\hline
\end{tabular}

$* p<.05 ; * * p<.01 ; * * * p<.001$

\section{Discusión}

Los resultados obtenidos aportan evidencia respecto de la relación entre la extraversión y la ansiedad y del neuroticismo con ansiedad y depresión en niños, de modo similar a otros resultados informados en la bibliografía (e.g. Ehrler \& Evans, 1999;
Vreeke \& Muris, 2012; Muris et al., 2004; Meesters et al., 2007). Contrariamente a lo esperado, no se observó una asociación entre la extraversión y la depresión. Esto resulta llamativo, ya que estudios en adultos (e.g. Malouff, Thorsteinsson \& Shutte, 2005) y también en niños (e.g. Agha-Yousefi \& Maleki, 2011) han informado relaciones en este 
sentido. Sin embargo, John et al. (1994) tampoco encontraron asociaciones de la extraversión con síntomas internalizantes en niños y adolescentes. Una explicación posible podría ser que al incluir el rasgo extraversión incluye varias facetas, entre las que se encuentran la presencia de emociones positivas y la sociabilidad (McCrae \& Costa, 1991), y si bien la falta de emociones positivas sería una característica de la depresión (anhedonia), no necesariamente sería una característica relacionada con otras dimensiones de la depresión como la menor sociabilidad o la preferencia por actividades solitarias. Por ejemplo, Hill y Argyle (2001) encontraron que la extraversión se asoció con la felicidad en participantes adultos; sin embargo, algunos de los participantes manifestaron, además, tener preferencia por la soledad, las actividades introspectivas y la relación con pocos amigos. De hecho, no se observaron diferencias en sus puntuaciones en felicidad con los individuos más extravertidos; por ello los autores denominaron a esta minoría los introvertidos felices (happy introverts). Coincidentemente, Naragon-Gainey, Watson y Markon (2009) encontraron que la depresión se asoció únicamente con la faceta emocionalidad positiva de la extraversión; en tanto otras facetas como sociabilidad, búsqueda de diversión y liderazgo solo presentaron asociaciones con la ansiedad social. En futuros estudios, podría resultar valioso analizar la contribución específica y diferencial de las distintas facetas de los rasgos de personalidad a la propensión a presentar síntomas clínicos.

Los resultados obtenidos también aportan evidencia respecto del rol mediador de la regulación emocional de modo similar a otros estudios realizados en adultos (e. g. Wang et al., 2009; Yoon et al., 2013). El análisis de los modelos permitió observar una influencia positiva de la extraversión en la RC, que a su vez influyó negativamente en la ansiedad. Otros estudios en niños y adolescentes también encontraron que la extraversión se ha asociado con la RC (e.g. Gresham \& Gullone, 2012; Gullone \& Taffe, 2011). El rasgo extraversión se caracteriza por varias facetas, como sensibilidad a la recompensa, emociones positivas, sociabilidad, asertividad y mucha energía (McCrae \& John, 1992), y se ha sugerido que la faceta relativa a las emociones positivas, especialmente el optimismo, podría favorecer la implementación de la RC (Carver \& Connor Smith, 2010). Las emociones positivas se relacionan con mayor capacidad para encontrar aspectos positivos en situaciones estresantes (Isen, 1990) y vuelven a los individuos más capaces de implementar recursos de afrontamiento efectivos para experiencias negativas (Fredickson, 2001), ya que las transforma de amenazantes en manejables.

A su vez, la $\mathrm{RC}$ ha resultado eficiente para la reducción del afecto negativo (Urry, 2009). Como sostienen Ray, McRae, Ochsner y Gross (2010), si las evaluaciones o appraisals son críticas en la generación de una emoción, la reevaluación cognitiva, al modificar o alterar estos appraisals, debería provocar un cambio en la respuesta emocional. Los niños con mayor frecuencia de uso de la RC han presentado menor cantidad de síntomas clínicos (Garnefski et al., 2007; Gullone \& Taffe, 2011).

En síntesis, es posible que la característica de emociones positivas del rasgo extraversión facilite la implementación de estrategias que requieren flexibilidad de pensamiento como la $\mathrm{RC}$ y, a su vez, la RC, al permitir una nueva evaluación de los eventos haciéndolos más benignos, permita disminuir las respuestas emocionales displacenteras como la ansiedad.

En cuanto a la depresión, los resultados obtenidos mostraron correlaciones bivariadas entre la $\mathrm{RC}$ y los síntomas de depresión, lo cual indica que la RC se asocia con menores niveles de depresión. Sin embargo, cuando la RC y la SEE se introducen simultáneamente en el modelo SEM, solamente la RC se vuelve un mediador significativo. Esto podría indicar que en la propensión a la psicopatología la presencia de estrategias disfuncionales es un factor de mayor peso que la falta de uso de estrategias adaptativas. Esta línea argumental está 
de acuerdo con el metanálisis realizado por Aldao, Nolen-Hoeksema y Schweizer (2010) y los análisis de mediación de Yoon et al. (2013). Aldao et al. (2010) demostraron que estrategias no adaptativas de regulación emocional — como rumiación, evitación, supresión del pensamiento y SEE — se asociaron más fuertemente con la psicopatología que las formas adaptativas de regulación emocional. El estudio de Yoon et al. (2013) analizó el efecto mediador de la $\mathrm{RC}$ y de estrategias no adaptativas —rumiación, supresión del pensamiento y SEEen la relación del neuroticismo con la depresión en población adulta. Sus resultados mostraron que si bien la RC correlacionó con la depresión en sentido negativo, al ser introducida en el modelo mediacional simultáneamente a las estrategias no adaptativas, no mostró un efecto mediador significativo y las estrategias no adaptativas tuvieron un efecto mediador total en esta relación.

Por último, el neuroticismo se asoció con la SEE y esta, a su vez, con mayores niveles de sintomatología clínica en ambos modelos y continuó ejerciendo un efecto directo en la ansiedad y depresión, más allá de este efecto indirecto. Los estudios en niños y adolescentes ya mencionados, de Gresham y Gullone (2011) y de Gullone y Taffe (2011), también encontraron que las puntuaciones en neuroticismo se asociaron con mayor frecuencia de uso de SEE. El neuroticismo incluye las facetas ansiedad, enojo-hostilidad, depresión, autoconciencia, vulnerabilidad e impulsividad (Costa \& McCrae, 1992), y es posible que los niños con neuroticismo en grado elevado perciban que la expresión de sus emociones negativas les dificulta la relación y aceptación de parte de sus pares, por lo que podrían optar por la SEE como forma de regular su expresión. En niños y adolescentes se ha registrado que la mayor frecuencia de uso de la SEE del enojo (no de tristeza) se asoció con síntomas internalizantes (Zeman, Shipman \& Suveg, 2002).

En síntesis, es posible que los individuos con altas puntuaciones en neuroticismo se esfuercen por controlar la expresión de sus emociones negativas para proteger las interacciones personales, lo cual se traduce en un aumento de los síntomas de ansiedad y depresión.

Finalmente, se observó una relación directa del neuroticismo en las variables clínicas, lo que estaría indicando que otros aspectos del neuroticismo que no guardan relación con la SEE se asocian con ansiedad y depresión, por ejemplo, el apoyo social. Existe evidencia de que el neuroticismo predice bajos niveles de apoyo social (Kendler, Gardner \& Prescott, 2006). De hecho, se ha encontrado que el apoyo social media parcialmente la relación entre el neuroticismo y la depresión (Finch \& Graziano, 2001; Kendler et al., 2006). Esta puede ser una explicación de los efectos directos del neuroticismo en la ansiedad y depresión que hemos observado.

Nuestro estudio presenta ciertas limitaciones. Por un lado, no es posible realizar atribuciones causales dadas la naturaleza transversal y correlacional del diseño. Por otro lado, debe tenerse en cuenta que existe la posibilidad de que muchas de las relaciones que se plantearon entre las variables sean relaciones bidireccionales. Si bien otros estudios presentan modelos con la misma direccionalidad que el nuestro, en el sentido de que los rasgos de personalidad preceden a los indicadores de bienestar (e. g. Mirnics et al., 2013; Yoon et al., 2013; Wang et al., 2009), sería adecuado llevar a cabo estudios longitudinales para entender mejor las posibles relaciones causales y bidireccionales. Además, es importante considerar que nuestros resultados surgen exclusivamente de cuestionarios de autoinforme, por lo que al trabajar con variables clínicas en niños podría ser enriquecedor utilizar otras fuentes (por ejemplo, entrevistas). Finalmente, al constituirse la muestra de manera intencional con determinadas características socioculturales, las posibilidades de generalización de los resultados son moderadas.

No obstante esto, los resultados de este trabajo permiten aumentar la evidencia disponible respecto de las relaciones entre los rasgos de personalidad 
y la propensión a presentar sintomatología clínica, así como que estas relaciones pueden estar mediadas por estrategias de regulación emocional. Si se considera que los rasgos de personalidad son relativamente estables y difíciles de modificar (Yoon et al., 2013) y que constituyen predictores importantes asociados a la ansiedad y depresión (Contrada, et al., 1990; Friedman, 1990), resulta valioso conocer los mecanismos a través de los cuales se ejerce su efecto en la sintomatología para la conducción de la prevención y la planificación de tratamientos (Bagby et al., 2008; Quilty et al., 2008). Estos mecanismos de regulación emocional son en sí mismos modificables o entrenables (e.g. Baumeister et al., 2006; MacLeod, Koster \& Fox, 2009), lo que constituye una vía de impacto para el diseño y contenido de programas de salud mental infantil (Garnefski et al., 2007). Sumado a esto, nuestros resultados aportan evidencia a la noción de que la regulación emocional podría constituirse en un proceso transdiagnóstico (Kring \& Sloan, 2010), lo cual permite una mayor comprensión de los procesos de comorbilidad entre los síntomas y favorece el desarrollo de protocolos de intervención unificados dirigidos al alivio y a la prevención de los síntomas (Aldao, 2012).

En este sentido, se han desarrollado protocolos unificados de intervención para favorecer la regulación emocional que se han mostrado efectivos para reducir tanto la ansiedad como la depresión (Ehrenreich-May, Kennedy \& Remmes, 2015; Suveg, Davis \& Jones, 2015). Por ejemplo, el Protocolo Unificado para el Tratamiento de los Trastornos Emocionales para Niños de 7 a 12 años de edad denominado Detectives Emoción (Ehrenreich-May $\&$ Bilek, 2012) tiene en cuenta la alta comorbilidad existente entre ansiedad y depresión y sus objetivos son brindar psicoeducación sobre el proceso emocional, practicar la exposición a situaciones emocionales y la aceptación de las experiencias emocionales displacenteras, enseñar a cuestionar los propios pensamientos, ofrecer entrenamiento para incrementar estrategias de regulación emo- cional como la $\mathrm{RC}$, disminuir estrategias como la evitación y, finalmente, prevenir de recaídas.

Nuestros resultados pueden constituir un complemento al diseño y contenido de estas intervenciones. En primer lugar, dada la asociación del rasgo extraversión con la RC, cobra valor promover el desarrollo de emociones positivas en los niños. Se ha mostrado que las emociones positivas influyen en el desarrollo de competencias personales, potencian la salud y la resiliencia psicológica y favorecerían la implementación de la RC (Fredrickson, 2000); por lo que se ha reconocido la importancia no solo de su promoción en contextos terapéuticos (Suveg, Hoffman, Zeman $\&$ Thomassin, 2009), sino en contextos escolares (Oros, Manucci \& Richaud de Minzi, 2011). En segundo lugar, nuestros resultados ponen en evidencia que podría resultar valioso prevenir el uso de estrategias como la SEE. Otros programas, como el Protocolo Unificado para el Tratamiento de los Trastornos Emocionales para Adolescentes (Ehrenreich, Goldstein, Wright \& Barlow, 2009), consideran explícitamente la necesidad de su prevención y, en este sentido, nuestro trabajo muestra que esta estrategia también es utilizada por niños de edad escolar y se asocia a consecuencias contraproducentes para su salud.

Finalmente, conocer que los rasgos de personalidad influyen en la sintomatología resulta de utilidad para guiar la prevención y planificar tratamientos (Bagby et al., 2008; Quilty et al., 2008), por lo que futuros estudios podrían analizar si los rasgos de personalidad poseen un rol moderador en la implementación de programas para la disminución de sintomatología; lo que representaría una contribución tanto para ampliar el conocimiento sobre estos procesos como para aumentar la mayor especificidad en el diseño de las intervenciones. Por último, la exploración de las variables de este estudio en población no clínica brinda insumos para implementar programas en contextos no clínicos (por ejemplo, el contexto escolar). El contexto escolar, como ámbito de intervención, comienza 
a reconocerse como sumamente valioso, ya que las intervenciones realizadas en este mejoran el funcionamiento emocional y comportamental de los niños, lo que reobra positivamente en el desempeño académico y la salud mental (Weist \& Murray, 2007).

\section{Referencias}

Agha-Yousefi, A. R. \& Maleki, B. (2011). Prediction of depression symptons by personality traits in children. Journal of Clinical Psychology, 3(1), 9-17

Aldao, A. (2012). Emotion regulation strategies as transdiagnostic processes: A closer look at the invariance of their form and function. Revista de Psicopatología y Psicología Clínica, 17(3), 261-277.

Aldao, A., Nolen-Hoeksema, S. \& Schweizer, S. (2010). Emotion-regulation strategies across psychopathology: A meta-analytic review. Clinical Psychology Review, 30, 217-237.

Bagby, R. M., Quilty, L. C., Segal, Z. V., McBride, C. C., Kennedy, S. H., \& Costa, P. T. Jr. (2008). Personality and differential treatment response in major depression: A randomized controlled trial comparing cognitive behavioural therapy and pharmacotherapy. Canadian Journal of Psychiatry, 53, 361-370.

Baumeister, R. F., Gailliot, M., DeWall, C. N., \& Oaten, M. (2006). Self- regulation and personality: How interventions increase regulatory success, and how depletion moderates the effects of traits on behavior. Journal of Personality, 74, 1773-1801.

Bentler, P. (1995). Structural equations program manual. Encino, CA: Multivariate Software.

Bienvenu, O., Samuels, J., Reti, I., Costa, P., Eaton, W. \& Nestadt, G. (2004). Anxiety and depressive disorders and the five-factor model of personality. Depression and Anxiety, 20, 92-97.

Bolger, N. \& Zuckerman, A. (1995). A framework for studying personality in the stress process.
Personality Processes and Individual Differences, 5, 890-902.

Bragado, C., Bersabé, R. \& Carrasco, I. (1999). Factores de riesgo para los trastornos conductuales, de ansiedad, depresivos y de eliminación en niños y adolescentes. Psicothema, 11(4), 939-956.

Brown, T. A. (2007). Temporal course and structural relationships among dimensions of temperament and DSM-IV anxiety and mood disorder constructs. Journal of Abnormal Psychology, 116, 313-328.

Brown, T. A., Chorpita, B. F., \& Barlow, D. H. (1998). Structural relationships among dimensions of the DSMIV anxiety and mood disorders and dimensions of negative affect, positive affect, and autonomic arousal. Journal of Abnormal Psychology, 107, 179-192.

Carver, C. S. \& Connor-Smith, J. K. (2010). Personality and coping. Annual Review of Psychology, 61, 679-704.

Carver, C. S., \& Scheier, M. F. (1998). On the self-regulation of behavior. New York: Cambridge University Press.

Casey, R. (1996). Emotional competence in children with externalizing and internalizing disorders. En M. Lewis \& M. Wolan (Eds.), Emotional development in atypical children (pp. 161-184). New York: Lawrence Erlbaum.

Contrada, R. J., Leventhal, H. \& O’Leary, A. (1990). Personality and heatlh. In L. A. Pervin (Ed.), Handbook of personality (pp. 638-670). New York: The Guilford Press.

Costa Jr., P., McCrae, R., \& Martin, T. (2008). Incipient adult personality: The -PI-3 in middle-school-aged children. British Journal of Developmental Psychology, 26(1), 71-89.

Costa, P. T., \& McCrae, R. R. (1992). Normal personality assessment in clinical practice: The NEO Personality Inventory. Psychological Assessment, 4, 5-13.

Coyne, J. C. (1976). Toward an interactional description of depression. Psychiatry, 39, 28-40. 
Diener, E. (1984). Subjective well-being. Psychological Bulletin, 95(3), 542-575.

Ehrenreich-May, J., \& Bilek, E. L. (2012). The Development of a Transdiagnostic, Cognitive Behavioral Group Intervention for Childhood Anxiety Disorders and Co-Occurring Depression Symptoms. Cognitive and Behavioral Practice, 19(1), 41-55.

Ehrenreich, J. T., Goldstein, C. M., Wright, L. R., \& Barlow, D. H. (2009). Development of a Unified Protocol for the treatment of emotional disorders in youth. Child \& Family Behavior Therapy, 31, 20-37.

Ehrenreich-May, J., Kennedy, S. M., \& Remmes, C. S. (2015). Emotion regulation interventions and childhood depression. In C. Timothy (Ed.), Self-regulated learning interventions with atriskyouth: Enhancing adaptability, performance, and well-being. (pp. 157-179). Washington, DC: American Psychological Association.

Ehrler, D. J., \& Evans, G. (1999). Extending big five theory into childhood: A preliminary investigation into the relationship between Big Five personality traits and behavior problems in children. Psychology in the Schools, 36(6), 451-458.

Finch, J. F., \& Graziano, W. G. (2001). Predicting depression from temperament, personality, and patterns of social relations. Journal of Personality, 69(1), 27-55.

Fredrickson, B. L. (2000, march 7). Cultivating positive emotions to optimize health and well-being. Prevention \& Treatment, 3, Article 0001a.

Fredrickson, B. L. (2001). The role of positive emotions in positive psychology: The broaden-andbuild theory of positive emotions. American Psychologist, 56, 218-226.

Friedman, H. S. (1990). Personality and disease. New York: Wiley.

Garnefski, N., Legerstee, J., Kraaij, V., van den Kommer, T., \& Teerds, J. (2002). Cognitive coping strategies and symptoms of depression and anxiety: A comparison between adolescents and adults. Journal of Adolescence, 25, 603-611.
Garnefski, N., Rieffe, C., Jellesma, F., Meerum Terwogt, M., \& Kraaij, V. (2007). Cognitive emotion regulation strategies and emotional problems in 9-11-year-old children: The development of an instrument. European Child \& Adolescent Psychiatry, 16, 1-9.

George, D., \& Mallery, P. (2011). SPSS for Windows step by step: a simple guide and reference (18.0 updated.). Boston, MA: Allyn \& Bacon/ Pearson.

Gerrig, R., \& Zimbardo, P. G. (2002). Psychology and life (16th ed.). Boston: Allyn \& Bacon.

Goodyer, I. M. \& Sharp, C. (2005). Child depression. En B. Hopkins, R. G. Barr, G. F. Michel y P. Rochat (Eds.), Child development (pp. 420424). Cambridge: Cambridge University Press.

Gresham, D., \& Gullone, E. (2012). Emotion regulation strategy use in children and adolescents: The explanatory roles of personality and attachment. Personality and Individual Differences, 52(5), 616-621.

Gross, J. \& John, O. (2003). Individual differences in two emotional regulation process: Implications for affect, relation-ships, and well-being. Journal of Personality and Social Psychology, 85, 348-362.

Gross, J.J. \& Thompson, R.A. (2007). Emotion regulation: Conceptual foundations. In J. J. Gross (Ed.), Handbook of emotion regulation. New York: Guilford Press.

Gullone, E. \& Taffe, J. (2011). The Emotion Regulation Questionnaire for Children and Adolescents: a psychometric evaluation. Psychological Assessment, 24(2), 409-417.

Harter, S., \& Nowakowski, M. (1987). Manual for the dimensions of depression profile for children and adolescents. Denver, CO: University of Denver.

Hills, P. \& Argyle, M. (2001). Happiness, introversión-extraversion and happy introverts. Personality and Individual Differences, 30(4), 595608. 
Hollingshead, A. B. (2011). Four factor index of social status. Yale Journal of Sociology, 8, 2-52.

Hughes, E. K., Gullone, E., Dudley, A. \& Tonge, B. (2010). A case-control study of emotion regulation and school refusal in children and adolescents. Journal of Early Adolescence, 30(5), 691-706.

Isen, A. M. (1990). Adaptation: Sociobiology, evolutionary psychology, and the variety of lessons to be learned. Motivation and Emotion, 14(4), 295-302.

John, O. \& Gross, J. (2004). Healthy and unhealthy emotion regulation: Personality processes, individual differences and life-span development. Journal of Personality, 72, 1301-33.

John, O. P., Caspi, A., Robins, R. W., Moffitt, T. E. \& Stouthamer-Loeber, M. (1994). The 'Little Five': exploring the nomological network of the five-factor model of personality in adolescent boys. Child Development, 65, 160-178.

Kendler, K. S., Gardner, C. O., \& Prescott, C. A. (2006). Toward a comprehensive developmental model for major depression in men. American Journal of Psychiatry, 163, 115-124.

Kline, R. B. (2011). Principles and practice of structural equation modeling. New York: Guilford Press.

Kring, A. M. \& Sloan, D. S. (2010). Emotion Regulation and Psychopathology. New York: Guilford Press.

Lahey, B. (2009). Public health significance of neuroticism. American Psychologist, 64(4), 241-256.

Lemos, V. (2006). La evaluación de la personalidad infantil a partir del enfoque de los cinco grandes factores de personalidad (Big Five). Psicodiagnosticar ADEIP, 16, 97-108.

Lewis, A. R., Zinbarg, R. E., \& Durbin, C. E. (2010). Advances, problems, and challenges in the study of emotion regulation: A commentary. Journal of Psychopathology and Behavioral Assessment, 32, 83-91.

MacKinnon, D. F. \& Hoehn-Saric, R. (2003). Is there a common etiology for depression and anxiety?
In K. Siegfried, A. Johan \& J. M. Sitse (Eds.), Handbook of depression and anxiety (pp. 111125). New York: CRC Press.

MacKinnon, D. P., Lockwood, C. M., \& Williams, J. (2004). Confidence limits for the indirect effect: Distribution of the product and resampling methods. Multivariate Behavioral Research, 39, 99-128.

MacLeod, C., Koster, E. H. W., \& Fox, E. (2009). Whither cognitive bias modification research? Journal of Abnormal Psychology, 118, 89-99.

Malouff, J. M., Thorsteinsson, E. B., \& Schutte, N. S. (2005). The relationship between the five-factor model of personality and symptoms of clinical disorders: A meta-analysis. Journal of Psychopathology and Behavioral Assessment, 27, 101-114.

Mardia, K. V. (1970). Measures of multivariate skewness and kurtosis with applications. Biometrika, 57, 519-530.

McCrae, R. R., \& Costa, P. T., Jr. (1991). The NEO Personality Inventory: Using the five-factor model in counseling. Journal of Counseling and Development, 69, 367-372.

McCrae, R.R. \& John, O.P. (1992). Introduction to the five-factor model and its applications. Journal of Personality, 60, 175-215.

Meesters, C., Muris, P., \& Van Rooijen, B. (2007). Reactive and regulative temperament factors and child psychopathology: Relations of neuroticism and attentional control with symptoms of anxiety and aggression. Journal of Psychopathology and Behavioral Assessment, 29, 149-158.

Merikangas, K. R. \& Angst, J. (1995). The challenge of depressive disorders in adolescence. En M. Rutter (Ed.), Psychosocial disturbances in young people (pp. 131-165). Cambridge: Cambridge University Press.

Mirnics, Z., Heincz, O., Bagdy, G., Surányi, Z., Gonda, X., Benko, ... Juhasz, G. (2013). The relationship between the big five personality dimensions and acute psychopathology: mediating 
and moderating effects of coping strategies. Psychiatria Danubina, 25(4), 379-388.

Mischel, W., Zeiss, R., \& Zeiss, A. (1974). Internal-external control and persistence: Validation and implications of the Stanford Preschool Internal-External Scale. Journal of Personality and Social Psychology, 29(2), 265-278.

Muris, P., De Jong, P. J. \& Engelen, S. (2004). Relationshiops between neuroticism, attentional control and anxiety disorders symtoms in non-clinical children. Personality and Individual Differences, 37, 789-797.

Naragon-Gainey, K.. Watson, D. \& Markon, K. E. (2009). Differential relations of depression and social anxiety symptoms to the facets of extraversion/positive emotionality. Journal of Abnormal Psychology, 118(2), 299-310.

$\mathrm{Ng}, \mathrm{W} . \&$ Diener, E. (2009). Personality differences in emotions: Does emotion regulation play a role? Journal of Individual Diferences, 30, 100-106.

Oros, L. B., Manucci, V. \& Richaud-de Minzi, M. C. (2011). Desarrollo de emociones positivas en la niñez. Lineamientos para la intervención escolar. Educación y Educadores, 14(3), 493-509.

Pascual, L., Galperín, C. Z. \& Bornstein, M. H. (1993). La medición del nivel socioeconómico y la psicología evolutiva: el caso argentino. Revista Interamericana de Psicología, 27(1), 59-74.

Penza-Clyve, C. \& Zeman, J. (2002). Initial validation of the Emotional Expression Scale for Children (EESC). Journal of Clinical Child and Adolescent Psychology, 31(4), 540-547.

Quilty, L. C., De Fruyt, F., Rolland, J.-P., Kennedy, S. H., Rouillon, P. F., \& Bagby, R. M. (2008). Dimensional personality traits and treatment outcome in patients with major depressive disorder. Journal of Affective Disorders, 108, 241-250.

Ray, R. D., McRae, K., Ochsner, K. N., \& Gross, J. J. (2010). Cognitive reappraisal of negative affect: Converging evidence from EMG and self-report. Emotion, 10, 587-592.

Richaud de Minzi, M. C., Sacchi, C., \& Moreno, J. E. (2001). Tipos de influencia parental, socialización y afrontamiento de la amenaza en la infancia [Informe 04-301, 1-25]. Subsidio otorgado por ANPCyT y CONICET. Buenos Aires, Argentina.

Sautú, R. (1989). Teoría y técnica en la medición del status ocupacional: escalas objetivas de prestigio [documento de trabajo]. Buenos Aires: Instituto de Ciencias Sociales de la Universidad de Buenos Aires.

Seisdedos Cubero, N. (1998). Cuestionario de Ansiedad Estado Rasgo: adaptación española. Madrid: Tea.

Smith, D. (1995). Towards explaining patterns and trends in youth crime. En M. Rutter (Ed.), Psychosocial disturbances in young people (pp. 166-211). Cambridge: Cambridge University Press.

Spielberger, C. D. (1973). Manual for the state-trait anxiety inventory for children. Palo Alto, CA: Consulting Psychologists Press.

Suveg, C., Davis, M., \& Jones, A. (2015). Emotion regulation interventions for youth with anxiety disorders. In C. Timothy (Ed.), Self-regulated learning interventions with at-risk youth: Enhancing adaptability, performance, and well-being (pp. 137-156). Washington D.C.: American Psychological Association.

Suveg, C., Hoffman, B., Zeman, J., \& Thomassin, K. (2009). Common and specific emotion-related predictors of anxious and depressive symptoms in youth. Child Psychiatry and Human Development, 40, 223-239.

Tabachnick, B. G., \& Fidell, L. S. (2001). Using multivariate statistics (4th ed.). Boston, MA: Allyn and Bacon.

Thompson, R. A. (1994). Emotion regulation: A theme in search of definition. Monographs of the Society for Research in Child Development, $59,25-52$

Urry, H. L. (2009). Using reappraisal to regulate unpleasant emotional episodes: Goals and timing matter. Emotion, 9, 782-797. 
Vreeke, L., \& Muris, P. (2002). Relations between inhibition, Big Five personality factors, and anxiety symptoms in non-clinical and clinically anxious children. Child Psychiatry and Human Development, 43(6), 884-894.

Wang, L., Shi, Z., \& Li, H. (2009). Neuroticism, extraversion, emotion regulation, negative affect and positive affect: the mediating roles of reappraisal and suppression. Social Behavior and Personality, 37(2), 193-194.

Watson, D., Clark, L. A., \& Harkness, A. R. (1994). Structures of personality and their relevance to psychopathology. Journal of Abnormal Psychology, 103, 18-31.

\section{Fecha de recepción: 6 de agosto de 2014} fecha de aceptación: 20 de mayo de 2015
Weist, M. D., \& Murray, M. (2007). Advancing school mental health promotion globally. Advances in School Mental Health Promotion, 1, 2-12.

Yoon, L. K., Maltby, J., \& Joormann, J. (2013). A Pathway from Neuroticism to Depression: Examining the Role of Emotion Regulation. Anxiety, Stress, and Coping, 26, 558-572.

Zeman, J., Shipman, K. \& Suveg, C. (2002). Anger and sadness regulation: predictions to internalizing and externalizing symptoms in children. Journal of Clinical and Adolescent Psychology, 31(3), 393-398. 
